\title{
Performance Improvement in MIMO-OFDM using BCH Coding and Interleaving
}

\author{
Anjali Kafaltiya \\ Uttarakhand Technical University \\ DIT Dehradun \\ India
}

\author{
P S Sharma \\ Dit University \\ DIT Dehradun \\ India
}

\begin{abstract}
In this paper, the performance of $\mathrm{BCH}$ correcting code is evaluated in comparison with Convolutional encoding using interleaved OFDM modulation. The system shows a consistent improvement in BER performance when add $\mathrm{BCH}$ coding on AWGN channel of OFDM with modulation schemes of 16 QAM. Hence the proposed MIMO-OFDM system has a better performance in terms of BER as compared to others modulation schemes.
\end{abstract}

\section{Keywords}

AWGN; BCH Coding; BER; DPSK; MIMO; OFDM; PSK; QAM; SNR.

\section{INTRODUCTION}

Advances in wireless communications, such as MIMO and OFDM provide the infrastructure for high spectral efficiency and consequently, high data rate. OFDM has become a popular technique for the transmission of signals [2]. Multiple transmit and receive antennas can be used to form multiple-input multiple-output (MIMO) channels, which provides considerable performance gain over SISO system. Even though the use of multiple antennas at the transmitter and receiver, can achieve higher data rates, but this is not always a preferred solution due to the size limitations at the mobile station. High order modulations such as 16 and 64-QAM can be used to increase the data rate of transmitter. To achieve improved performance for MIMO system, it is also important to design a receiver structure which can detect and decode the signal received through MIMO channels. Here there are different modulation schemes such as DPSK, PSK and 16-QAM which can fulfil the requirements of different applications.

In OFDM, transmission is carried out in parallel on different frequencies. That is, the entire channel is divided into many narrow band sub channels, which are transmitted in parallel, thereby, increasing the symbol duration and reducing the ISI. The carrier spacing is selected such that the modulated carriers are orthogonal over a symbol interval. In addition, a guard interval (cyclic prefix) is inserted in order to combat the frequency effective technique for combining multipath fading and high bit rate transmission [6]. MIMO communication techniques have received great attention and gained significant development in recent years. This is due to the fact that a MIMO channel can offer a significant capacity gain over a traditional SISO channel [13] [14]. This paper evaluates the performance of DPSK, PSK and 16-QAM in MIMO-OFDM subjected to AWGN channel. Here two different encoding schemes are used. The analysis has been performed by using MATLAB for simulation and evaluation of BER for both modulation techniques and analysis which modulation gives better result in convolution and $\mathrm{BCH}$ encoding.

\section{SYSTEM DESCRIPTION}

In this paper, an interleaved coded transmission is proposed with different modulation schemes for MIMO-OFDM system. A coded OFDM system is considered with 64 data subcarriers and 16-QAM constellation. Matrix interleavers are used, the cyclic prefix length is said to the channel maximum delay. It is now accepted that multiple input multiple output (MIMO) system increases link reliability and spectral efficiency of multiuser wireless communication [5]. The paper deals with the BER performance evaluation of DPSK, PSK and 16-QAM modulation schemes for $\mathrm{BCH}$ and Convolutionally coded system over AWGN channel. The performance of different modulation techniques are analyzed in terms of BER and SNR. Based on simulation results it can be concluded that the 16-QAM gives better gain in SNR performance as compared to DPSK and PSK. Hence the performance of different modulation schemes namely, DPSK, PSK and 16-QAM is evaluated.

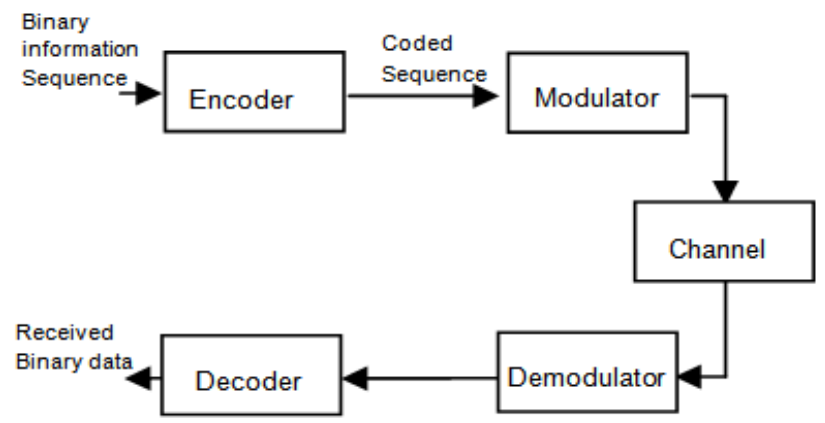

Fig.1. General Block diagram of communication system

OFDM scheme is being used in modern communication system as it is spectrally efficient. In this scheme guard interval is inserted in the time domain using cyclic prefix to achieve error free communication, as the ISI gets mitigated between OFDM symbols. The OFDM guard interval can be inserted by the cyclic extensions of the OFDM symbol with cyclic prefix (CP) or cyclic suffix (CS) [9]. AWGN is a noise that affects the transmitted signal when it passes through the channel. It contains a uniform continuous frequency spectrum over a particular frequency band. Here $\mathrm{BCH}$ and Convolutional coding are opted as one knows [7]-[8], it is a powerful error correcting code that can correct both random and bursty errors. 


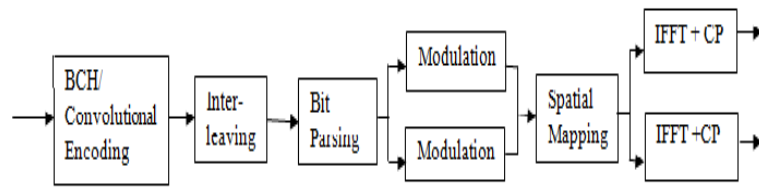

(a)

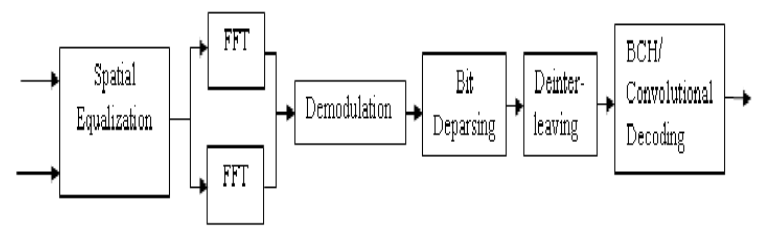

(b)

Fig.2. (a) Transmitter and (b) Receiver processing for the MIMO-OFDM system with Forward Error Correction

This section defines MIMO-OFDM system model as summarized in the above figure which represents the communication procedure in MIMO-OFDM.

\section{(a) INTERLEAVING}

A primary technique which is effective in overcoming error bursts, is interleaving. Interleaving is a standard signal processing technique used in a variety of communication system. The classical use for interleaving is to disperse sequences of bits in a bit stream so as to minimize the effect of burst errors introduced in transmission and the BER reduces at the output [10]. This has become an extremely useful technique for minimization of errors. The basic work done by an interleaver is that it rearranges the order of symbols to be transmitted following a particular rule and at the receiver the reverse rule can be used to restore the original sequence.

There are two classical kinds of interleavers, commonly referred to as Block and Convolutional. In this paper the model is motivated by the matrix interleaver, as it is one type of block interleaver.

In describing the OFDM system matrix interleavers are used here. The matrix interleaver performs block interleaving by filling a matrix with the input symbols row by row and then sending the matrix contents to the output port column by column [11].

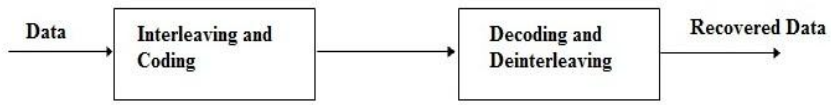

Fig.3. Interleaving

Matrix Interleaving: Conventionally, interleaving is used to spread out the errors occurring in bursts like those exhibited in fading channels [10]. In this regard, matrix interleaving where bits are fed in a matrix row by row and read out column by column, is usually implemented. At the transmitter, the interleaver is used to feed the OFDM symbols with different permutations of the information sequence so that the generated parity sequences can be assumed independent. At the receiver, the deinterleaver is used to randomize the symbols after every decoding step, thus making the iterative decoding more efficient [18]. In this paper, it is attempted to provide an in-depth study on the performance of OFDM with matrix interleaver. Such an interleaver is completely defined by $n$ and $\mathrm{m}$ and is thus referred to as (n, m) matrix interleaver. The column size as $\mathrm{n}$ and the row size as $\mathrm{m}$. at the deinterleaver, information is within column wise and read out row wise. The capability of burst error scattering for the matrix interleaver depends on the values of $\mathrm{n}$ and $\mathrm{m}$.

\section{(b) CODING TECHNIQUES}

Channel coding represents the source information over the channel in a manner that minimizes the error probability in decoding by adding the redundant bits systematically. Channel coding is more important over the wireless channels; the bit error rate gets reduced at final reception. Channel coding in general can be done by errordetecting or error correcting codes [15].

There are two basic approaches of adding structured redundancy used for controlling errors-

\section{(a) Automatic Repeat Request (ARQ)}

(b) Forward Error Correction (FEC)

ARQ utilizes parity bits or redundant bits for checksum added to the data to detect an error. The receiving terminal does not attempt to correct the error always; rather it requests the transmitter to retransmit the data. Hence a two way link is required between transmitter and receiver. This detection process sends an acknowledgement (ACK) or not acknowledgement (NAK) back to the transmitting node to request retransmission.

FEC uses redundant bits added systematically to detect and correct the error independently. These schemes do not send ACK/NAK signals. Here only a one-way link is sufficient for proper communication, error handling is totally done by the receiver.

Basically there are two types of codes in general:

- $\quad$ Error-Detecting Codes: - These kinds of codes are basically like, parity check, LRC-VRC check, and CRC check.

- Error-Correcting Codes:- These are mainly used in wireless communication and are categorized as follows:

\section{Block Codes:}

(i) Hamming code

(ii) Bose-Chaudhary-Hocquenghem $(\mathrm{BCH})$ codes

(iii) Reed-Solomon codes

II. Convolutional Codes:

III. Turbo Codes:

(i) Block/ product turbo codes (BTC or PTC)

(ii) Convolutional turbo codes (CTC)

On further explaining the proposed coding techniques i.e. Convolutional and $\mathrm{BCH}$ codes it is seen that $\mathrm{BCH}$ coding gives better performance.

BCH Coding: One of the most important and powerful classes of linear block codes are $\mathrm{BCH}$ codes, and it contains the following parameters: 
Block Length: $n=2^{m}-1$

Message Size: k symbols

Parity Check Size: $r=(n-k)=2 t_{c}$ symbols

Minimum Distance: $d_{\min }=2 t_{c}+1$ symbols

No. of correctable symbols in error: $t_{c}=(n-k) / 2$

During code design, there is a precise control over the number of symbol errors correctable by the code. It corrects multiple bit errors and can be easily decoded using syndrome decoding. [16] $\mathrm{BCH}$ codes are the random error correcting codes and these are flexible while choosing the code parameters like block length and code rate. [19]

Convolutional Coding: The major application of Convolutional codes is that they are used to improve the performance of wireless links and are used in most of latest mobile networks. A Convolutional encoder is called so because it performs a convolution of the input stream with the encoder's impulse responses. $\mathrm{M}$ bit information symbol to be encoded is transformed into an $\mathrm{n}$-bit symbol, where $\mathrm{m} / \mathrm{n}$ is the code rate. Basically used to achieve a reliable data transfer, these are more powerful for error correction than block codes.

\section{SIMULATION RESULTS}

This paper analyses the bit error rate performance of Convolutional and BCH code in AWGN channel using DPSK, PSK and 16-QAM as modulation schemes. The BCH encoder block creates a $\mathrm{BCH}$ code with constraint length $\mathrm{K}=7$. Here a coded OFDM system with 64 subcarriers $(\mathrm{N}=64)$ and the number of constellations for QAM are 16 i.e. 16-QAM is considered. Matrix interleaver is used and cyclic prefix is set at $25 \%$ (16). There are two transmit and receive antennas. The figure (4-9) shows the BER Vs SNR for different systems using different modulation schemes.

It is even observed from figure that BER keeps improving on using BCH coding for DPSK, PSK, and 16-QAM respectively. A "waterfall" like sudden drop of the bit error rate (BER) can be commonly observed at low-to-moderate signal-to-noise ratio's (SNR). The proposed system achieves both coding and diversity gain, scheme improved with Convolutional encoder, and the proposed $\mathrm{BCH}$ encoder achieves better error-correcting performance. The Forward Error Correction (FEC) block includes encoding and interleaving. In the above used schemes, the input data is first encoded using Convolutional or $\mathrm{BCH}$ encoder followed by puncturing. The next step involved is interleaving using a matrix interleaver. The receiver then performs the same functions in reverse order.

Table 1. Parameters Used

\begin{tabular}{|l|l|}
\hline Parameters & Values \\
\hline Modulation Techniqu & DPSK,PSK,16-QAM \\
\hline Coding & BCH/ Convolutional \\
\hline Interleaver & Matrix \\
\hline Data Subcarriers & 64 \\
\hline Cyclic Prefix & 16 \\
\hline System & OFDM \\
\hline
\end{tabular}

\begin{tabular}{|l|l|}
\hline $\begin{array}{l}\text { MIMO Tx \& } \\
\text { Antennas }\end{array}$ & $2 \mathrm{x} 2$ \\
\hline Type of Channel & AWGN \\
\hline
\end{tabular}

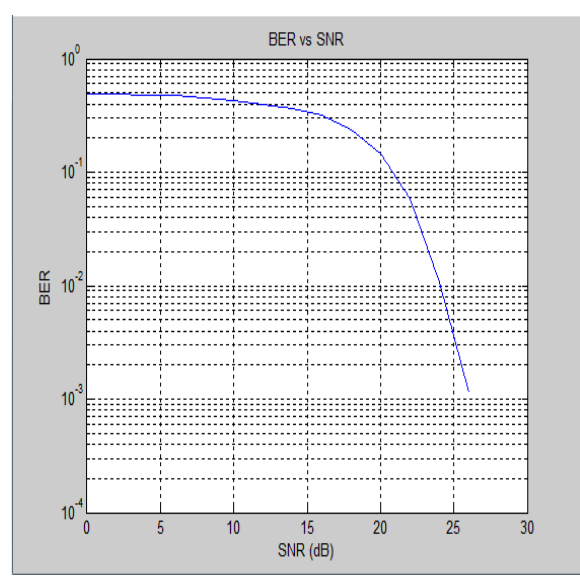

Fig.4. BER vs. SNR for Convolutionally coded DPSK System

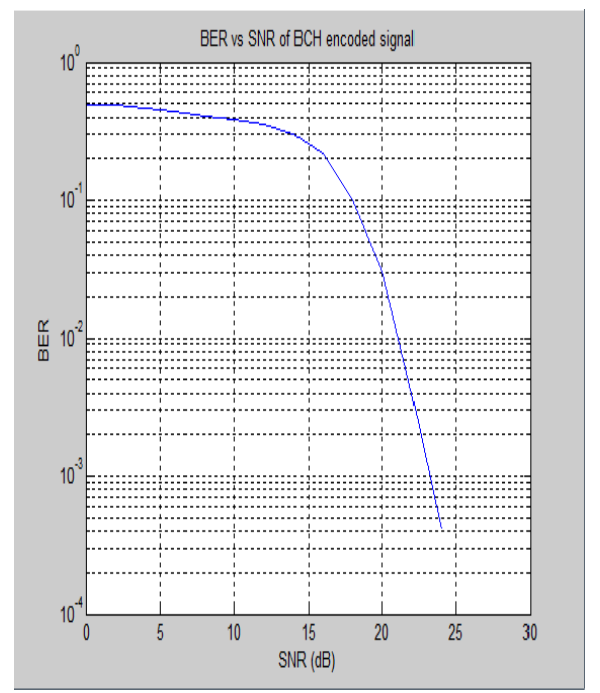

Fig.5. BER vs. SNR for BCH coded DPSK System

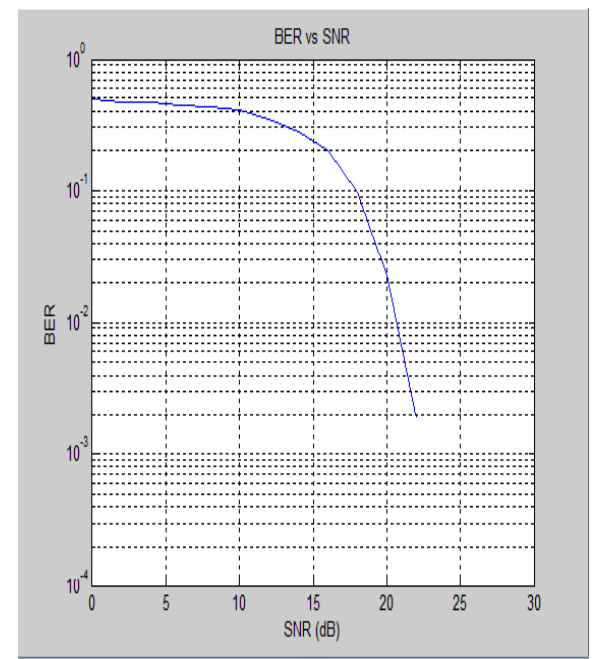

Fig.6. BER vs. SNR for Convolutionally coded PSK System 


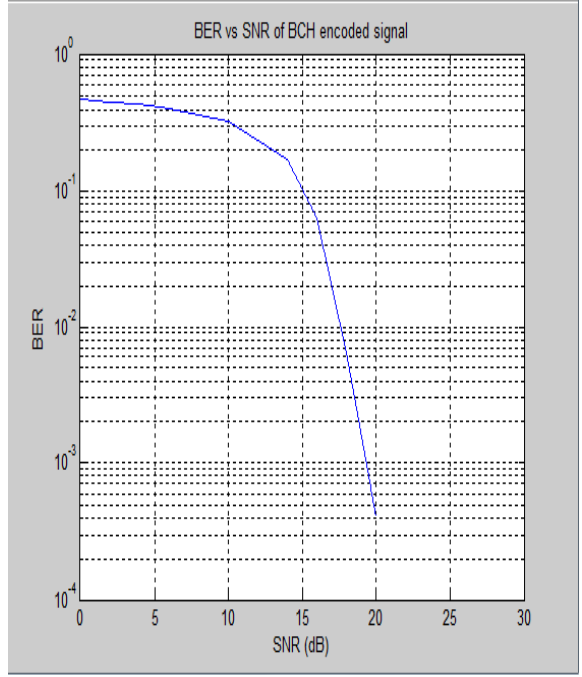

Fig.7. BER vs. SNR for BCH coded PSK System

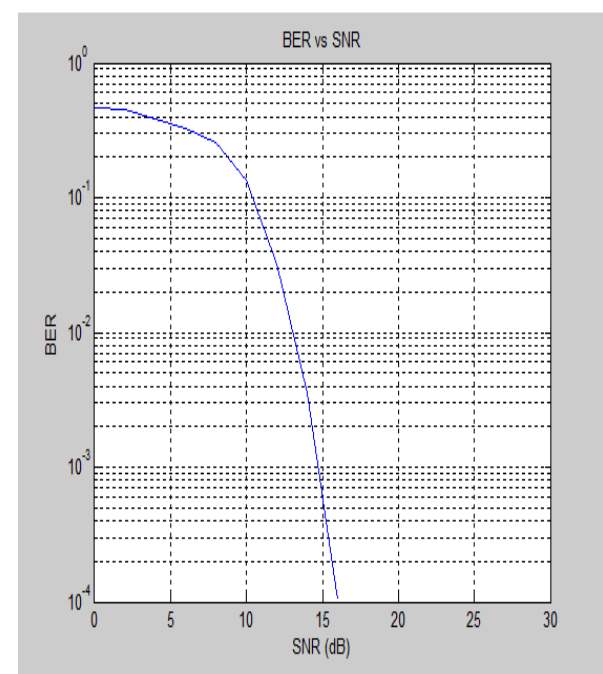

Fig.8. BER vs. SNR for Convolutionally coded QAM System

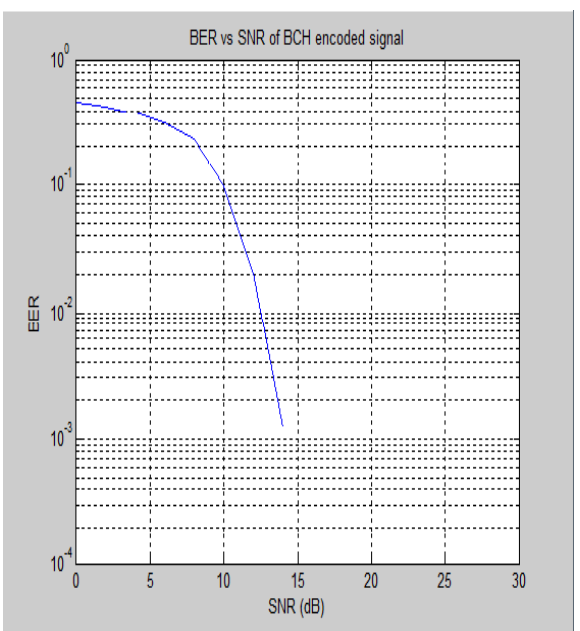

Fig.9. BER vs. SNR for BCH coded QAM System

\section{CONCLUSION}

Here the simulation results show the comparison analysis of PSK, DPSK and 16-QAM modulation schemes over AWGN channel. Based on the simulation results it is concluded that by using 16QAM scheme better SNR performance for same value of BER is obtained as Compared to the PSK and DPSK. Through simulation the BER performance of the system and the minimum required SNR to satisfy both high quality and low quality of data services is obtained.

\section{FUTURE SCOPE}

The work presented in this paper, i.e. the performance of PSK, DPSK and 16-QAM for OFDM system can be extended to multicarrier code division multiple access (MC-CDMA) system. The results drawn from this paper can also be extended to include the performance of PSK, DPSK and 16-QAM over other generalized fading channels.

\section{REFERENCES}

[1] Robert C. Daniels, Constantine M. Caramanis and Robert W. Heath, Members IEEE, "Adaptation in convolutionally coded MIMO-OFDM wireless systems through supervised learning and SNR ordering". IEEE Transactions on vehicular Technology, January, 2010, Vol. 59, No. 1.

[2] R. Prasad, OFDM for wireless communication system, Artech House, 2004.

[3] Chan- Ho Choi and Gi-Hong, Sr. member IEEE, "Bit Interleaved Coded Transmission with Multilevel", IEEE GLOBE COM, 2009.

[4] Yi-Min Lin, Hsie-Chia Chang, and Chen-Yi Lee, "Improved High Code rate soft $\mathrm{BCH}$ decoder architectures with one extra error compensation", IEEE Transactions on very large scale integration(VLSI) systems, Nov 2013, Vol. 21, No. 11.

[5] G. Foschini and M. Gans, "On limits of wireless communications in a fading environment when using multiple antennas," wireless personal communications, March 1998, vol. 6, pp. 311-335.

[6] Jae-Min Kwak, Sung-Chul Lee, Ji-Woong Kim, Gi-Sik, Sung-Eon Cho and Hyo-Chang Pang, "The performance simulation of OFDM system using hierarchical 16 QAM on multipath fading channel", Journal of Security Engineering, Nov 2005, Vol. 2 No.1.

[7] K H H Wong, L. hanzo and R. Steele, "Channel coding for satellite mobile channels," Int. J satellite Communication, 1989, Vol, 7, pp. 143-163.

[8] L. Hanzo, K H H Wang, and R Steele, "Efficient Channel Coding and Interleaving schemes for mobile radio communications" in proc, Inst. Elec, Eng, Colloquium on Microcellular Mobile Radio, Feb, 22 1989, London, Savoy Place.

[9] Xiaojing Huang, "Effect of DC offset on OFDM system with zero padded suffix". IEEE, 2006.

[10] F. Rey, M. Lamarca and G. Vazquez, "Adaptive Interleaver based on rate- compatible punctured Convolutional codes", IEEE Transactions on communications, June 2009, Vol-57, No. 6. 
[11] MATLAB, "HELP, Communication, toolbox, Block Interleaving".

[12] Vineet Chaturvedi, Vivek Kumar Gupta, DIT Dehradun, "Performance Analysis for Different Interleavers in Various Modulation Schemes with OFDM over an AWGN Channel", IOSR Journal of Engineers. April, 2012, Vol (2(4)) pp. 760-767.

[13] C N Chuah, DNC Tse, J M Khan, and R A Valenzuela, "Capacity scaling in MIMO wireless system under correlated fading, "IEEE Transactions on Information Theory, Vol. 48, No. 3, pp. 637-650, 2002.

[14] P F Driessen and G. J Foschini, "On the capacity formula for multiple input multiple output wireless channels: A Geometric Interpretation," IEEE Transactions Communications Feburary 1999. Vol. 2, pp. 173-176.

[15] Upena Dalal, Electronics Engineering Department,Sardar Vallabhbhai National Institute of Technology, Surat, "Wireless Communication", (C) Oxford University Press 2009.

[16] Z. Iqbal et al, "Analysis and Design of Coding and Interleaving in a MIMO OFDM Communication
System," IEEE Transactions on Consumer Electronics, August 2012, Vol. 58, No. 3.

[17] Hun Hee Lee; Myung Sun Baek; Tec Hoon Kim; Hyoung Kyu Song; "Efficient detection scheme in MIMO-OFDM for high speed wireless home network system", IEEE Trans Consumer Electronics, May 2009, Vol 55, No. 2, pp 507-572

[18] Zhen-dong Zhang, Bin Wu, Yong-Xu Zhu, Yu-mei Zhou, "Design and implementation of a Multi-Mode Interleaver/Deinterleaver for MIMO OFDM Systems" IEEE 2009.

[19] Junho Cho and Wonyong Sung, Senior Member , IEEE, " Efficient Software- Based Encoding and Decoding of BCH Codes" IEEE Transactions On Computer s, July 2009, Vol. 58, No. 7

[20] Suzi Seroja Sarnin, Nani Fadzlina Naim, wan Nor Syafizan W. Muhamad, "Performance Evaluation Of Phase Shift Keying Modulation Technique Using BCH Code, Cyclic Code and Hamming Code Through AWGN Channel Model in Communication System". 